\title{
Comparison of oral hygiene status of young adult population of age group 20-25 years in the urban and rural areas
}

\author{
Randhir Kumar, ${ }^{1, *}$, Geeta Sharma² \\ 1,2Professor \& HOD, ${ }^{1}$ Dept of Periodontology and Implantology, ${ }^{2}$ Dept of Oral Pathology, ${ }^{1}$ Patna Dental College Patna, Bihar, \\ ${ }^{2}$ Sarjug Dental College Darbhanga, Bihar, India
}

*Corresponding Author:

Email: drrandhirmds@gmail.com

\begin{abstract}
Oral hygiene status is very important, it shows person's attitude towards the oral hygiene and also oral cavity is first entry for food and its digestion. The presence of food debris and calculus on tooth surface reflects the oral hygiene status of the person.

Objectives of the Study: To observe the oral hygiene status of young adult of age group of 20-25 years in the rural and urban area of Patna.

Materials and Methods: Cross sectional descriptive study of rural and urban population of 20-25 years of age.

Results: The inter group variation in OHI-S is very small and not significant in the urban populations. The inference of oral hygiene status of rural population is showing that comes under poor oral hygiene status while the OHI-S of urban area comes under fair oral hygiene status but it is on higher side of it.
\end{abstract}

Keywords: Oral hygiene, OHI, Urban, Rural.

\section{Introduction}

Oral hygiene status is very important, it shows person's attitude towards the oral hygiene and also oral cavity is first entry for food and its digestion. The presence of food debris and calculus on tooth surface reflects the oral hygiene status of the person. These calculus, food debris and plaque are main etiological factors for periodontal destruction. The periodontium acts as the supporting and investing structure of tooth. So the disease of periodontium resulted as loss of supporting and investing structure. The poor oral hygiene is also one of the main etiological factors for halitosis i.e. bad breath. Good oral hygiene is the mirror of the personality. So the study on oral hygiene status of the person is very important to decide the necessity oral health education and treatment need of the population of particular area. Most of the studies on the oral hygiene status of the population were conducted in India either in rural areas or in urban areas but very few comparative studies are published. Again in India large populations are present in the age group 20-25. So this study is planned to conduct a comparative study on oral hygiene status of young adult population of age group 20-25 years in the urban and rural areas of Patna.

\section{Objectives of the Study}

1. To observe the oral hygiene status of young adult of age group of 20-25 years in the rural area of Patna.

2. To observe the oral hygiene status of young adult of age group of 20-25 years in the urban area of Patna.

3. To compare the oral hygiene status of young adult of age group of 20-25 years in the urban and rural areas of Patna.

\section{Review of Literature}

In 2006 Anil S Hari S and Vijay Kumar $\mathrm{T}^{3}$ assessed periodontal condition of urban and rural areas of Trivandrum and they found that oral hygiene status of rural area is poorer in rural areas than the urban areas. Maity A.K Banarjee K L and T K PAL ${ }^{4}$ (2007) examined 5960 subjects in rural population and found calculus was wide spread in most of the subjects. Mehta et al. ${ }^{1}$ (2008) conducted a study on oral hygiene status of rural population of Ratnagiri district of Maharashtra and found oral hygiene status was poor in this population. Loe et al. ${ }^{2}$ conducted a study to evaluate the oral hygiene status of Shri lankan tea workers and he found oral hygiene status is very poor among them. Kapoor et al. ${ }^{1}$ (2004) conducted a study to measure the oral hygiene status of adult population of 16-30 years of rural population around Lucknow. A bdellatif H.M. and

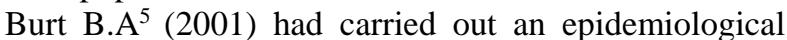
investigation into the relative importance of age and oral hygiene status on determinant of periodontitis and they found positive correlation between oral hygiene status and periodontitis. Nikias $\mathbf{M}^{2}$ in 2001 studied on oral disease and quality of life and found positive correlations.

\section{Methodology}

1. Study design- cross sectional descriptive.

2. Study area- urban and rural areas of Patna.

3. Study period-60days between.

4. Participant - Population between 20-25 years (no. of participant 600 ).

300 Subjects are selected randomly from the urban area and 300 are from rural area of Patna district. 


\section{Inclusion criteria:}

1. Those subject who never went to dental clinic or attended any oral and dental awareness camp. Those who come in the age group of 20-25 years of both the sex group

2. The person having at least incisors and first molars Exclusion criteria:

1. Age group less or more of this age group

2. Pregnant woman

3. Subjects having any known acute or chronic disease

All the subject who satisfied desired criteria will be examine for Simplified oral hygiene index by Green and Vermillion.

Surfaces and teeth to be examined

11- Upper right central incisor- labial surface

16- Upper right first molar- buccal surface

26- Upper left first molar- buccal surface

31-Lower left central incisor- labial surface
36- Lower left first molar- lingual surface 46- Lower right first molar- lingual surface It has two components

1. Calculus index $(0-3)$

2. Debris index $(0-3)$

OHI-S= CI-S + DI-S

The simplified oral hygiene index values ranges from 0 6

Good -0.1 t0 1.2

Fair - 1.3 to 3.0

Poor -3.1 to 6.0

Study Tools

1. Mouth mirror

2. Dental explorer

3. Performa

\section{Results}

\section{Periodontal Disease Status}

\begin{tabular}{|c|c|c|c|c|c|c|c|c|c|c|}
\hline \multirow{2}{*}{ Oral hygiene status } & & \multicolumn{3}{|c|}{ 20-21 Yrs Group R1 } & \multicolumn{3}{|c|}{ 22-23 Yrs Group R2 } & \multicolumn{3}{|c|}{ 24-25Yrs Group R3 } \\
\hline & & M & $\mathbf{F}$ & $\mathbf{T}$ & $\mathbf{M}$ & $\mathbf{F}$ & $\mathbf{T}$ & M & $\mathbf{F}$ & $\mathbf{T}$ \\
\hline State rural & $\mathrm{N}=$ & 21 & 23 & 44 & 49 & 36 & 85 & 51 & 20 & 71 \\
\hline Mean Calculus index-S & & 2.9 & 2.5 & 2.7 & 2.6 & 2.4 & 2.5 & 2.5 & 2.7 & 2.6 \\
\hline $\begin{array}{l}\text { Mean Debris } \\
\text { index-S }\end{array}$ & & 2.3 & 2.1 & 2.2 & 2.4 & 2.2 & 2.3 & 2.6 & 2.4 & 2.5 \\
\hline \multirow[t]{2}{*}{ Mean OHI-S } & & 5.2 & 4.6 & 4.9 & 5.0 & 4.6 & 4.8 & 5.1 & 5.1 & 5.1 \\
\hline & & \multicolumn{3}{|c|}{ U1 } & \multicolumn{3}{|c|}{$\mathrm{U} 2$} & \multicolumn{3}{|c|}{ U3 } \\
\hline Status urban & $\mathrm{N}=$ & 30 & 9 & 39 & 46 & 32 & 78 & 48 & 35 & 83 \\
\hline Mean Calculus index-S & & 1.6 & 0.9 & 1.25 & 1.6 & 1.9 & 1.75 & 1.1 & 1.4 & 1.25 \\
\hline $\begin{array}{l}\text { Mean Debris } \\
\text { index-S }\end{array}$ & & 1.2 & 1.7 & 1.45 & 1.2 & 1.5 & 1.35 & 1.4 & 1.2 & 1.3 \\
\hline Mean OHI-S & & 2.8 & 2.6 & 2.7 & 2.8 & 3.4 & 3.1 & 2.5 & 2.6 & 2.55 \\
\hline
\end{tabular}

$\mathrm{R}=$ Rural $\mathrm{U}=$ Urban

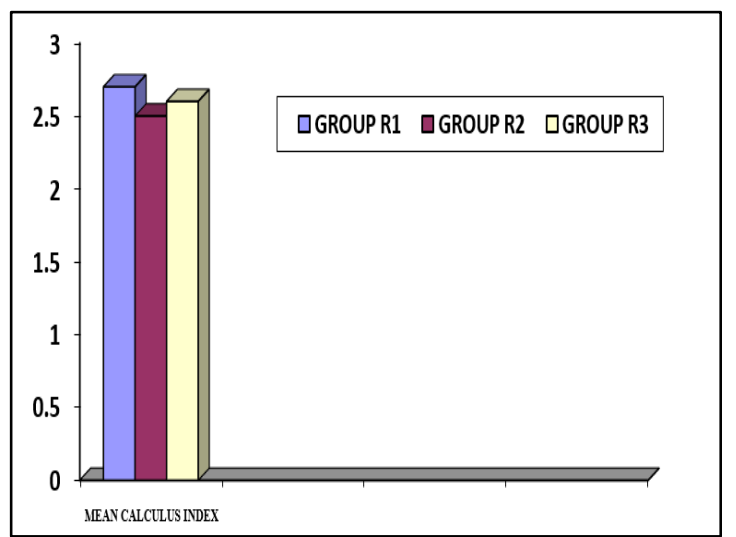

Fig. 1: Shows relationship between mean calculus index of group R1, R2, R3, in rural population

The mean calculus index of rural population shows its variation from 2.4 to 2.9 in different age group but they do not show any particular correlation between these age groups. The difference were not statistically significant

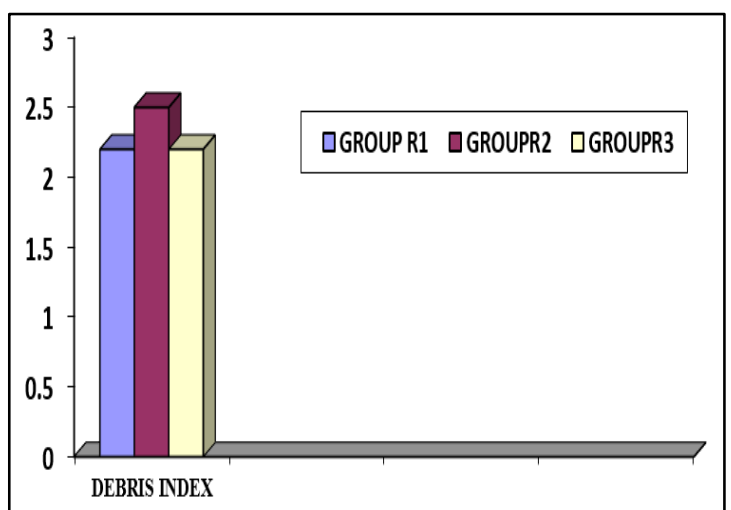

Fig. 2: Shows Relationship between mean debris index of group R1, R2, R3 In rural population

The mean debris index of rural population shows its variation from 2.1 to 2.6 in different age group but they do not show any particular correlation between these age groups. The difference were not statistically significant. 


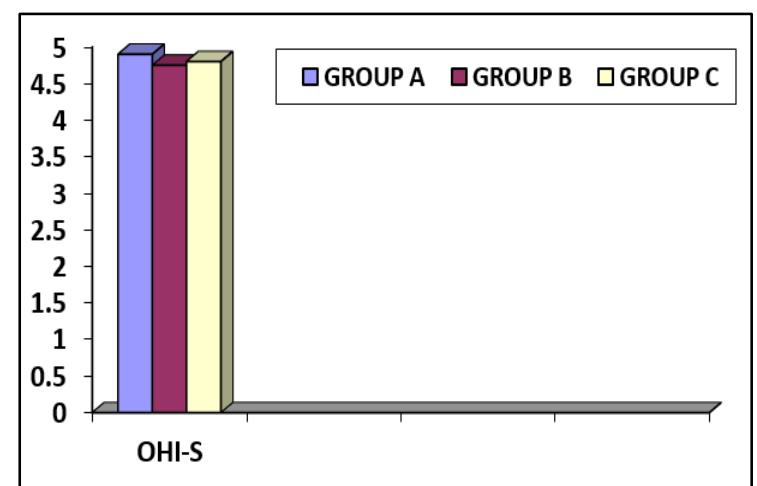

Fig 3: Shows relationship between mean OHI-S of group R1, R2, R3 in rural population

The Mean Oral Hygiene Index-S of rural population shows its variation from 4.6 to 5.1 in different age group but they do not show any particular correlation between these age groups. The difference were not statistically significant. The interpretation of Mean Oral Hygiene Index of rural population shows that these population have oral hygiene status is poor.

Graph1: Shows comparison of OHI status of urban and rural population

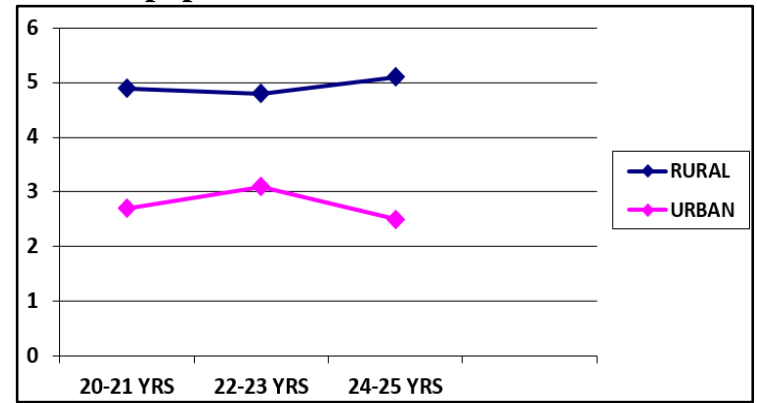

The variation within the group either urban or rural is very small. The interpretation of the oral hygiene status of rural area population is poor while the oral hygiene status of urban area is fare.

\section{Discussion}

The results of the present study showed mean CI index (MCI) was 2.7 in the age group of 20-21yrs 2.5 in $22-23 y r s$ age group and 2.6 in age group of $24-25 y r s$ in rural population. The inter group variation in MCI is very small and not significant in the rural populations. The mean debris index (MDI) was 2.3 in the age group of 20-21yrs, 2.3 in 22-23yrs age group and 2.5 in age group of 24-25yrs in rural population. The inter group variation in MDI is very small and not significant in the rural populations. Mean Oral Hygiene Index (OHI-S) was 4.9 in the age group of $20-21 \mathrm{yrs} 4.8$ in $22-23 \mathrm{yrs}$ age group and 5.1 in age group of 24-25yrs in rural population. The inter group variation in OHI-S is very small and not significant in the rural populations. The mean CI index (MCI) was 1.25 in the age group of 20$21 \mathrm{yrs}, 1.75$ in 22-23yrs age group and 1.25 in age group of 24-25yrs in urban population. The inter group variation in MCI is very small and not significant in the urban populations. The mean debris index (MDI) was 2.3 in the age group of $20-21 \mathrm{yrs} 2.3$ in $22-23 \mathrm{yrs}$ age group and 2.5 in age group of 24-25yrs in urban population. The inter group variation in MDI is very small and not significant in the urban populations. Mean Oral Hygiene Index (OHI-S) was 2.7 in the age group of 20-21yrs 3.1 in 22-23yrs age group and 2.55 in age group of 24-25yrs in urban population. The inter group variation in OHI-S is very small and not significant in the urban populations. The inference of oral hygiene status of rural population is showing that comes under poor oral hygiene status while the OHI-S of urban area comes under fair oral hygiene status but it is on higher side of it. When simplified oral hygiene index of rural and urban population was compared, then it was observed that oral hygiene status of rural population was poorer than the urban population and the difference was statistically significant. The result of present study is coincide with the finding obtained by Anil S, Hari S and Vijay Kumar T (2006). CI observed in present study showed similar pattern as observed by Maity AK, Banarjee K L and T K PAL (2007).

\section{Conclusion}

The pattern of oral hygiene status was similar in both rural and urban areas. However oral hygiene status of rural population was poorer than the urban area. This may happens due to difference in method and frequency of teeth cleaning. In urban population means of teeth cleaning was tooth brush and tooth paste and some subjects clean their tooth twice daily while in rural tooth cleaning methods are with datoon, tooth powder and by finger are common and no one was cleaning their teeth twice daily.

In future for more precise result this study may be conducted on large population and some other marker for periodontal status like bleeding on probing, periodontal pocket, loss of attachment may be included as a marker for periodontal status.

\section{References}

1. Essentials of preventive and community dentistry Soben peter $4^{\text {th }}$ edition. National oral health survey. 2002-2003.

2. Anil S, Hari S and Vijay Kumar T. Assessment of periodontal conditions of adult population from urban and rural areas of trivendram using CPITN Index. JISP. 2006;3.

3. Maity AK, Banarjee K L and T K PAL, Periodontal status of farmers in rural population of West Bengal. J of Oral health. 2007;2.

4. Carranza FA and Newman M G. 10th Edition Clinical Periodontology.

5. Vimal Sikri and Poonam Sikri; $2^{\text {nd }}$ edition Community dentistry.

6. Ainamo J; Significance of epidemiologic research in the understanding of periodontal disease. Scand. J Dent Res. 1992;39:100. 
7. Burke P. J. T. and Wilson; evaluation of oral health by general dental practioners; JDR. 1994;73.

8. Green J.C. and Vermillion; The simplified oral hygiene index. J Am Dent Assoc. 1964;68.

9. Johansen J R and Hangen E; The need of periodontal reatment in urban population; J Clin Period. 1985;2:26.

How to cite this article: Kumar R, Sharma G. Comparison of oral hygiene status of young adult population of age group 20-25 years in the urban and rural areas. Int J Periodontol Implantol. 2018;3(4):124127. 